\title{
El pH como parámetro en la síntesis de hidroxiapatita y cloroapatita a partir del método hidrotermal asistido por microondas
}

\section{$\mathrm{pH}$ as a parameter in the synthesis of hydroxyapatite and chloroapatite from the microwave-assisted hydrothermal method}

\author{
L.S. Villaseñor-Cerón ${ }^{1}$, M. I. Reyes-Valderrama ${ }^{(D a}$, S. López-Ortiz ${ }^{(D a}$, E. Salinas-Rodríguez ${ }^{(\mathbb{D} a}$, \\ V. Rodríguez-Lugo ${ }^{\mathrm{a}, *}$ \\ a Area Académica de Ciencia de la Tierra y Materiales, Universidad Autónoma del Estado de Hidalgo, 42184, Pachuca, Hidalgo, México.
}

\section{Resumen}

En el presente trabajo se realiza la síntesis de apatitas, a través del método hidrotermal asistido por microondas, variando el pH en 5, 6, 7, 8, 9 y 10. La caracterización fisicoquímica y estructural se realizó por las técnicas: MEB-EDS, DRX y FTIR, determinando un tamaño de cristal de 49 a $31 \mathrm{~nm}$ en función del pH; se observó la formación de aglomerados, constituidos por fibras de entre 43.4 a $108.5 \mathrm{~nm}$ de diámetro y 412.4 a $1603.8 \mathrm{~nm}$ de longitud, así mismo se determina la presencia de planos cristalográficos característicos de HAp y ClAp. Se determinó el tamaño de cristal, identificando un decremento del pH9 al pH5 de 49 a 31 nm, en consecuencia de emplear ácido nítrico para disminuirlo. Además, se identifican los modos vibracionales pertenecientes a ambas estructuras y una elongación en la banda del grupo $\mathrm{OH}^{-}$, atribuida a la interacción entre el grupo $\mathrm{OH}^{-}$de la $\mathrm{HAp}$ y los iones de $\mathrm{Cl}^{-}$de la $\mathrm{ClAp} \mathrm{Los}$ resultados obtenidos contribuyen a establecer el efecto del $\mathrm{pH}$ en el tamaño de cristal y de partícula para la síntesis de HAp y ClAp.

\section{Palabras Clave:}

Hidroxiapatita, Cloroapatita, pH, Tamaño de partícula, Morfología.

\begin{abstract}
In the present work the synthesis of apatite's is carried out, through the microwaves-assisted hydrothermal method, varying the $\mathrm{pH}$ at 5, 6, 7, 8, 9 and 10. The physicochemical and structural characterization was carried out by MEB-EDS, DRX and FTIR techniques, determining a crystal size from 49 to $31 \mathrm{~nm}$ depending on the $\mathrm{pH}$; it was observed the formation of agglomerates constituted by fibers between 43.4 and $108.5 \mathrm{~nm}$ in diameter and lengths from 412.4 to $1603.8 \mathrm{~nm}$. Presence of crystallographic planes characteristic of HAp and ClAp were identified, in which the crystal size was determined, showing a decrease from 49 to $31 \mathrm{~nm}$ for $\mathrm{pH} 9$ to $\mathrm{pH} 5$, respectively; this decrease is associated with the addition of the nitric acid in the synthesis process. The vibrational modes belonging to both structures and an elongation in the absorption band of the $\mathrm{OH}^{-}$group were identified, attributed to the interaction between the $\mathrm{OH}^{-}$group of the $\mathrm{HAp}$ and the $\mathrm{Cl}^{-}$ions of the ClAp. The results obtained contribute to establish the effect of $\mathrm{pH}$ on the crystal and particle size for the synthesis of HAp and ClAp.
\end{abstract}

Keywords:

Hydroxyapatite, Chloroapatite, $\mathrm{pH}$, Particle size, Morphology.

\section{Introducción}

Las apatitas son minerales que poseen una amplia gama de aplicaciones industriales, como los fertilizantes en la industria agrícola y el fósforo en la industria de la iluminación (Yanagisawa et al., 1999). Así mismo, pueden encontrarse biológicamente como: la hidroxiapatita $\left(\mathrm{Ca}_{10}\left(\mathrm{PO}_{4}\right)_{6}(\mathrm{OH})_{2}\right)$, la cual ha sido ampliamente estudiada en las últimas décadas, debido a que este material es el principal componente inorgánico en huesos y dientes de mamíferos y vertebrados (Mehdi Sadat-Shojai et al., 2013; Olszta et al., 2007). Además, la hidroxiapatita (Hap) presenta propiedades tales como; biocompatibilidad (Szcześ et al.,

\footnotetext{
*Autor para la correspondencia: ventura.r165@gmail.com (Susana López Ortiz), salinasr@uaeh.edu.mx (Eleazar Salinas Rodríguez) y ventura.rl65@gmail.com (Ventura Rodríguez Lugo)

Correo electrónico: leslysabina@gmail.com (Lesly Sabina Villaseñor Cerón), mariaisabelreyv@gmail.com (María Isabel Reyes Valderrama), multimediasusy@hotmail.com 
2017), osteointegración (Kusakabe et al., 2004), osteoinducción, baja o nula toxicidad, bioactividad (Chetty et al., 2012) y posee un alto potencial osteogenético, posicionándolo como un excelente biomaterial (Habibovic et al., 2008; Kokubo \& Takadama, 2006; Mehdi Sadat-Shojai et al., 2013; O’Hare et al., 2010; Rabiei et al., 2007).

Los minerales naturales de apatita biológica pueden intercambiar iones $\mathrm{OH}^{-}, \mathrm{F}^{-}, \mathrm{y} \mathrm{Cl}^{-}$en su estructura (Yanagisawa et al., 1999). Por lo que, la modificación de las apatitas sintéticas es de sumo interés, debido a que influye en sus propiedades químicas y físicas, así como su capacidad de unión ósea, tal es el ejemplo de la sustitución del ion cloruro en la apatita, que existe como un oligoelemento en la fase mineral del hueso y los dientes. El cloro puede incorporarse a la estructura de la HAp, reemplazando el grupo hidroxilo, adquiriendo el nombre de cloroapatita $\left(\mathrm{Ca}_{10}\left(\mathrm{PO}_{4}\right)_{6} \mathrm{Cl}_{2}\right)$; su importancia radica en que los iones cloruro tienen la capacidad para desarrollar un ambiente ácido en la superficie del hueso que activa los osteoclastos en el proceso de resorción ósea (Harlov et al., 2002; Kannan et al., 2007). Por otra parte, la cloroapatita (ClAp) y la HAp, se estudian para el desarrollo de materiales, con uso en la contención de desechos nucleares e industriales (Rulis et al., 2004). Así mismo la ClAp, ha sido estudiada para sintetizar un nuevo tipo de nanocloroapatita estabilizada (nClAp) para la remediación de sedimentos contaminados de $\mathrm{Pb}$ (II), Cd(II) y Cr(VI) (Deng et al., 2020; Han et al., 2021; Huang et al., 2018; Wan et al., 2018).

Debido a esto se han implementado diferentes rutas de síntesis para la obtención de HAp y ClAp, mismas que son clasificadas como: métodos secos (estado sólido, mecano químico), métodos húmedos (precipitación química, hidrólisis, sol-gel, hidrotermal, emulsión) y procesos a alta temperatura (pirólisis, combustión) (Ferraz et al., 2018; Koutsopoulos, 2002; Mehdi Sadat-Shojai et al., 2013; V. Rodríguez-Lugo et al., 2001; Sáenz et al., 2003; Zheo et al., 2014). También se han utilizado diferentes precursores como fuentes de calcio y de fosfato entre los que desatacan la Brushita, monetita, el fosfato octacálcico, el fosfato $\alpha$-tricálcico, el fosfato $\beta$-tricálcico, el fosfato monocálcico monohidratado y el fosfato anhídrido, con la finalidad de encontrar las condiciones óptimas para la obtención de HAp y ClAp (Recillas et al., 2003; V. Rodríguez-Lugo et al., 2003, 2012, 2015).

Otro punto fundamental para la obtención de HAp y ClAp, son las condiciones de síntesis, como: la concentración, la temperatura de reacción, tiempo de reacción y $\mathrm{pH}$, mismos que contribuyen a tener un mayor control del tamaño y forma de la partícula, lo que permite ampliar los campos de aplicaciones para estas apatitas (Ciobanu et al., 2013; López-Ortiz et al., 2020; Mehdi SadatShojai et al., 2013; V. Rodríguez-Lugo et al., 2018; SánchezCampos et al., 2021; Zuo et al., 2017). Estudios recientes, establecen que mantener un control en el tamaño y forma de partícula, y cristalinidad se ve reflejado en las propiedades químicas y físicas del mismo, presentando características como inhibidor de células cancerosas, así como en diferentes aplicaciones biomédicas e industriales destacando en estas últimas: los catalizadores para la adición de tipo Michael, materiales de acogida para láseres, materiales de fluorescencia, conductores iónicos, sensores de gas, entre otros. (Dorozhkin, 2010; Koutsopoulos, 2002; V.Rodríguez-Lugo et al., 2020).

El presente trabajo de investigación se enfoca en estudiar el efecto del $\mathrm{pH}$ sobre la obtención de HAp y ClAp, sintetizadas mediante el método hidrotermal asistido por microondas, empleando como precursores de calcio y fosfato, el cloruro de calcio $\left(\mathrm{CaCl}_{2}\right)$ y fosfato de sodio tribásico dodecahidratado $\left(\mathrm{Na}_{3} \mathrm{PO}_{4} \cdot 12 \mathrm{H}_{2} \mathrm{O}\right)$, respectivamente, modificando el $\mathrm{pH}$ para cada síntesis $(5,6,7,8,9,10)$.

\section{Desarrollo Experimental}

\subsection{Sintesis de Hap y ClAp}

La síntesis de la hidroxiapatita y cloroapatita se llevó a cabo mediante el método hidrotermal asistido por microondas, empleando como precursores cloruro de calcio y fosfato de sodio tribásico dodecahidratado, en condiciones estequiométricas de acuerdo con la ecuación 1 (Ec. 1):

$$
\begin{aligned}
& 10 \mathrm{CaCl}_{2}+6 \mathrm{Na}_{3} \mathrm{PO}_{4}+2 \mathrm{H}_{2} \mathrm{O} \stackrel{\Delta}{\rightarrow} \mathrm{Ca}_{5}\left(\mathrm{PO}_{4}\right)_{3} \mathrm{OH}+ \\
& \mathrm{Ca}_{5}\left(\mathrm{PO}_{4}\right)_{3} \mathrm{Cl}+18 \mathrm{NaCl}+\mathrm{HCl}+\mathrm{H}_{2} \mathrm{O} \ldots \ldots \ldots \ldots \ldots \ldots \text {. Ec. } 1
\end{aligned}
$$

El procedimiento consistió en diluir los precursores de $\mathrm{Ca}$ y $\mathrm{PO}_{4}{ }^{-3}$ en $10 \mathrm{~mL}$ de agua desionizada, a una agitación constante durante 10 minutos. Después se añadió la solución de calcio a la solución de fosfato manteniendo agitación constante, obteniendo una solución de $\mathrm{pH}$ igual a 9. Posteriormente se añadió por goteo hidróxido de amonio para aumentar el $\mathrm{pH}$ a 10 y ácido nítrico para disminuir el $\mathrm{pH}$ a 8, 7, 6 y 5. Subsecuentemente las muestras fueron trasladadas al equipo de microondas en donde se llevó a cabo la síntesis a $200^{\circ} \mathrm{C}$ durante $40 \mathrm{~min}$. Finalmente, las muestras fueron lavadas con agua desionizada, secadas en una estufa de baja temperatura a $100^{\circ} \mathrm{C}$ durante 24 horas y posteriormente fueron calcinadas a $450^{\circ} \mathrm{C}$ durante 3 horas.

\subsection{Caracterización}

Las muestras obtenidas fueron caracterizadas por Microscopia Electrónica de Barrido de Bajo Vacío (LV-SEM) utilizando un equipo JEOL modelo JMS 5900-LV, se montaron sobre un porta muestras de aluminio de $10 \mathrm{~mm}$ de diámetro, utilizando cinta adhesiva de carbón. La medición de las partículas se llevó a cabo empleando el software "ImageJ". El análisis cristalográfico se realizó en un difractómetro de rayos $\mathrm{X}$ marca SIEMENS, modelo D-5000, operado a $35 \mathrm{KV}, 25 \mathrm{~mA}$, con un intervalo de barrido de $10^{\circ}$ a $70^{\circ}$ en $2 \theta$ con un tiempo y tamaño de paso de 1 segundo y $0.03^{\circ}$, respectivamente. Finalmente, el análisis químico de las muestras se realizó en un espectrómetro infrarrojo modelo Nexus 670 FTIR E.S.P. Marca Nicolet. Cada muestra fue mezclada con bromuro de potasio $(\mathrm{KBr})$ para ser colocadas en la cámara del espectrómetro.

\section{Resultados}

\subsection{Microscopia Electrónica de Barrido}

Se realizó la caracterización microestructural de la hidroxiapatita y cloroapatita por MEB (Figura 1). Observando en la Figura 1a) pH5 una morfología constituida de fibras de tamaño promedio de $108.5 \mathrm{~nm}$ de diámetro y $1603.8 \mathrm{~nm}$ de longitud. En la Figura 1b) pH6 se ilustra la formación de fibras de $105.2 \mathrm{~nm}$ de diámetro y $822.7 \mathrm{~nm}$ de longitud, en el caso de la Figura 1c) pH7 se distingue la formación de aglomerados cuyas dimensiones son del orden de $2.3 \mu \mathrm{m}$ de diámetro y $4.6 \mu \mathrm{m}$ de longitud, mismas que se encuentran constituidas por fibras de $51.3 \mathrm{~nm}$ de diámetro y $489.7 \mathrm{~nm}$ de longitud, en la Figura 1d) pH8 se distinguen aglomerados de $4 \mu \mathrm{m}$ de diámetro y $10 \mu \mathrm{m}$ de longitud, constituidos por fibras de $665.5 \mathrm{~nm}$ de longitud y $105.7 \mathrm{~nm}$ de diámetro, en el caso de la Figura 1e) pH9 se aprecian aglomerados de $2 \mu \mathrm{m}$ de diámetro y $5 \mu \mathrm{m}$ de longitud, constituidos por fibras de $43.4 \mathrm{~nm}$ de diámetro y $412.4 \mathrm{~nm}$ de longitud, Finalmente, en la Figura 1f) pH10, se aprecian aglomerados de $5 \mu \mathrm{m}$ de diámetro y $7 \mu \mathrm{m}$ de longitud, constituidos por fibras de $72.6 \mathrm{~nm}$ de diámetro y $571.4 \mathrm{~nm}$ de longitud. 

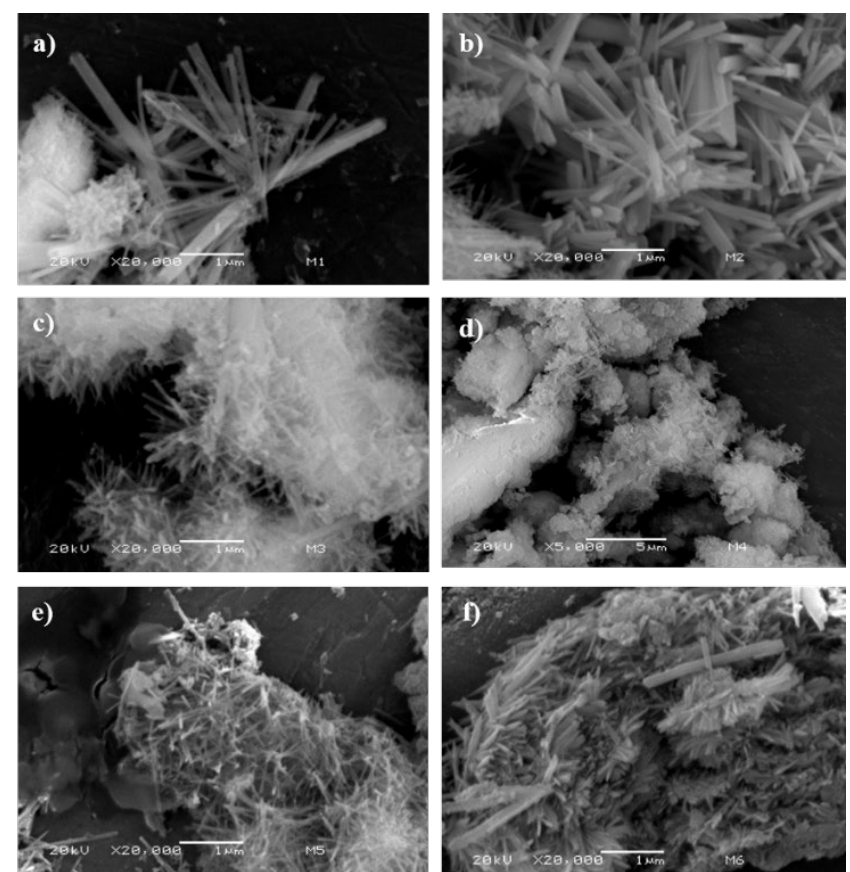

Figura 1. Micrografía de la HAp y ClAp a pH de: a) 5, b) 6, c) 7, d) 8, e) 9 y f) 10 .

En la Figura 2, se muestra la gráfica de los tamaños promedio de partícula correspondientes a cada muestra a $\mathrm{pH} 5, \mathrm{pH} 6, \mathrm{pH} 7$, pH8, pH9 y pH10, en donde se identifica el cambio de tamaño promedio de las dimensiones de longitud y diámetro. Se aprecia un incremento del pH5 al pH8, observando una disminución del tamaño de partícula en ambas dimensiones, teniendo en el último un incremento del tamaño de partícula en relación con los demás pH's, sin embargo, el aumento del $\mathrm{pH} 9$ al $\mathrm{pH} 10$, favorece un aumento del tamaño de partícula en la longitud y el diámetro de las fibras.

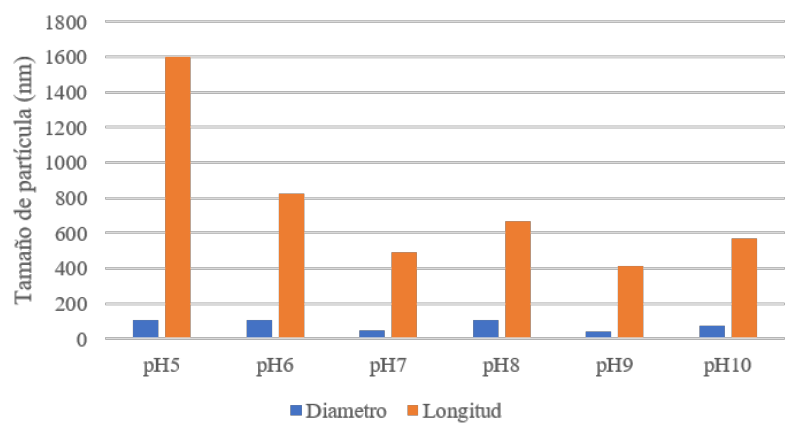

Figura 2. Gráfica en la que se identifica el tamaño promedio de partícula de HAp y CIAp a pH5, 6, 7, 8, 9 y 10 respectivamente.

En la Tabla 1, se incluye el análisis elemental EDS correspondientes a las muestras de $\mathrm{pH} 5, \mathrm{pH} 6, \mathrm{pH} 7, \mathrm{pH} 8, \mathrm{pH} 9$ y $\mathrm{pH} 10$, en la cual se identifica el porcentaje en peso (wt $\%)$ de los elementos de $\mathrm{Ca}, \mathrm{P}, \mathrm{Na}$ y $\mathrm{Cl}$; siendo los dos últimos, subproductos que corresponde a los precursores de fosfato y calcio, en donde este último contribuye a la formación de la cloroapatita, bajo las condiciones de síntesis empleadas. Por otra parte, estos resultados permiten obtener la relación de $\mathrm{Ca} / \mathrm{P}$ y Ca/Cl para cada muestra, apreciando un incremento en la relación de 2.8 a 4.39 para $\mathrm{pH}^{\prime} \mathrm{s}$ de 5 a 8; para $\mathrm{pH}^{\prime} \mathrm{s}$ de 9 y 10 disminuye a 3.55 y 3.37, respectivamente. En el caso de la relación de $\mathrm{Ca} / \mathrm{Cl}$, se observa un incremento de 7.97 a 10.04 para $\mathrm{pH} 5 \mathrm{a} \mathrm{pH7}$ y una disminución para $\mathrm{pH} 7$ a $\mathrm{pH} 9$ de 3.25 a 3.5 y a pH10 incrementa a 4.8 , como se apreciar en la Tabla 1.

Tabla 1: Análisis Elemental localizado por EDS.

\begin{tabular}{ccccccc} 
& \multicolumn{6}{c}{ Muestra (\% en peso) } \\
\cline { 2 - 7 } Elemento & $\mathbf{p H 5}$ & $\mathbf{p H 6}$ & $\mathbf{p H 7}$ & $\mathbf{p H 8}$ & $\mathbf{p H 9}$ & $\mathbf{p H 1 0}$ \\
$\mathbf{O}$ & 33.24 & 35.94 & 31.50 & 32.54 & 31.44 & 31.89 \\
$\mathbf{N a}$ & 0.57 & 0.74 & 3.33 & 2.41 & 3.01 & 1.57 \\
$\mathbf{P}$ & 15.92 & 15.06 & 12.45 & 9.84 & 11.79 & 13.13 \\
$\mathbf{C l}$ & 5.60 & 4.37 & 11.07 & 11.95 & 11.84 & 9.09 \\
$\mathbf{C a}$ & 44.66 & 43.90 & 36.01 & 43.24 & 41.92 & 44.32 \\
$\mathbf{C a} / \mathbf{P}$ & 2.80 & 2.91 & 2.89 & 4.39 & 3.55 & 3.37 \\
$\mathbf{C a} / \mathbf{C l}$ & 7.97 & 10.04 & 3.25 & 3.61 & 3.5 & 4.8 \\
\hline
\end{tabular}

\subsection{Difracción de Rayos $X$}

La caracterización de la estructura cristalina de las muestras obtenidas de la síntesis de HAp y ClAp se determinó mediante Difracción de rayos $X$. En la Figura 3 se observan los difractogramas de las muestras de HAp y ClAp, para los diferentes pH's. Para la HAp se identifican los picos correspondientes a la tarjeta PDF 70-0795 atribuida a la fase hexagonal, principalmente a $16.77^{\circ}, 25.98^{\circ}, 28.16^{\circ}, 32.64^{\circ}, 39.44^{\circ}, 43.91^{\circ}, 47.83^{\circ}, 54.54^{\circ}$, $57.10^{\circ}, 61.60^{\circ}, 64.44^{\circ}, 67.37^{\circ}$ y $69.20^{\circ}$ para 2-theta, que corresponden a la indexación (101), (201), (300), (130), (113), (104), (501), (124), (332), (224) y (105), respectivamente. Se identifican picos de difracción a $10.75^{\circ}, 16.77^{\circ}, 25.98^{\circ}, 28.16^{\circ}$, $31.70^{\circ}, 32.20^{\circ}, 39.13^{\circ}, 47.83,51.83^{\circ}$ y $55.11^{\circ}$ para 2 -theta; al hacer un búsqueda en la base datos de la ICDD (International Centre of Diffraction Data) se identifican los planos cristalográficos correspondientes a la tarjeta PDF 24-0214 de la fase monoclínica y con indexación (-201), (-211) y (-412), (-602), (-612), (221), (-1003), (-824), (-1023) característicos de la cloroapatita; asimismo se identifica un traslape en los picos con la fase de hidroxiapatita, la cual se forma al mismo tiempo que la fase de cloroapatita.

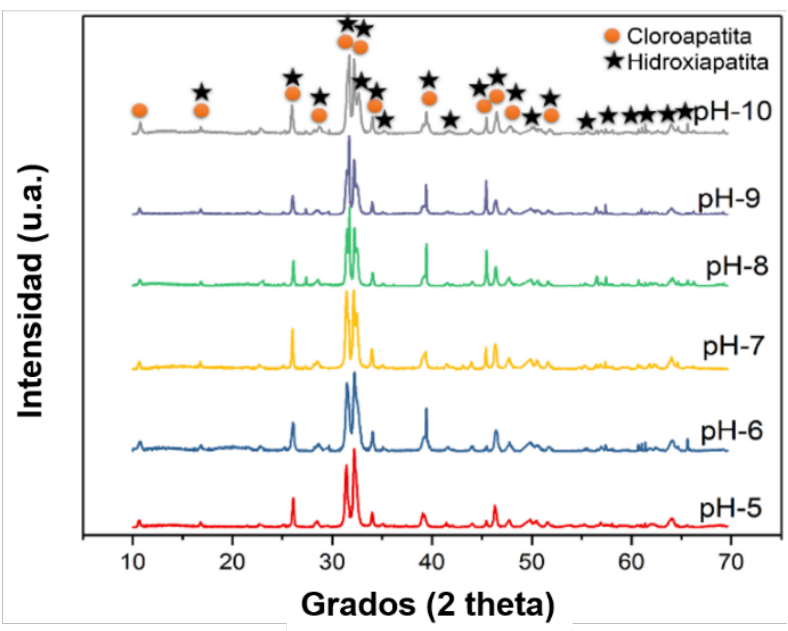

Figura 3. Difractograma de las muestras de CIAp y HAp a pH5, 6, 7, 8, 9 y 10 respectivamente. 
A partir de los planos cristalográficos principales se realizó el cálculo para obtener el tamaño promedio de cristal de acuerdo con la ecuación de Debye-Scherrer (Ec.2):

$$
\left(D=\frac{\kappa \cdot \lambda}{B \cdot \cos \theta}\right)
$$

En la Tabla 2 se visualiza el tamaño de cristal promedio para cada muestra, a diferente $\mathrm{pH}: 10,9,8,7,6$ y 5 , respectivamente. Donde se distingue que para un $\mathrm{pH}$ de 9 a 10 se observa una disminución del tamaño promedio de cristal de 49 a $47 \mathrm{~nm}$, respectivamente y para un $\mathrm{pH}$ de $9,8,7,6$ y 5 se aprecia una disminución de 49, 44, 42, 33 y $31 \mathrm{~nm}$, respectivamente.

\begin{tabular}{cc} 
Tabla 2: Tamaño de cristal de las muestras a pH 5, 6, 7, 8, 9 y 10. \\
\hline $\begin{array}{c}\text { TH de la muestra } \\
\text { HAp/ClAp }\end{array}$ & $\begin{array}{c}\text { Tamaño del } \\
\text { cristal (nm) }\end{array}$ \\
\hline $\mathbf{1 0}$ & 47 \\
$\mathbf{9}$ & 49 \\
$\mathbf{8}$ & 44 \\
$\mathbf{7}$ & 42 \\
$\mathbf{6}$ & 33 \\
$\mathbf{5}$ & 31 \\
\hline
\end{tabular}

\subsection{Espectroscopia Infrarroja por Transformada de Fourier}

Se empleó el análisis FTIR para evaluar la estructura química entre la HAp y ClAp a diferentes pH's. La Figura 4, muestra los espectros FTIR del HAp pura y su variación de pH's a 5, 6, 7, 8, 9 y 10 respectivamente.

El espectro FTIR muestra las bandas características de HAp, a $1045 \mathrm{~cm}^{-1}$ pertenecientes al modo vibracional de estiramiento asimétrica $\left(v_{3}\right)$, en $952 \mathrm{~cm}^{-1}$ se observa un modo vibracional de estiramiento simétrico $\left(v_{1}\right)$; las bandas en 616 y $562 \mathrm{~cm}^{-1}$ son atribuidas a un modo vibracional de flexión asimétrica $\left(v_{4}\right)$ correspondientes a el grupo $\mathrm{PO}_{4}^{-3}$ de la estructura. En la banda con un máximo de alrededor de $3429 \mathrm{~cm}^{-1}$ correspondiente al modo vibracional de estiramiento y en $1636 \mathrm{~cm}^{-1}$ se asigna a la banda con un modo vibracional de flexión simétrica $\left(v_{2}\right)$ pertenecientes al grupo $\mathrm{OH}^{-}$, del agua adsorbida presente en la hidroxiapatita (Panda et al., 2003). Asimismo, se observa una elongación en la banda de la región de las vibraciones del grupo $O H^{-}$, en 3640,3570 y $3498 \mathrm{~cm}^{-1}$, atribuidas a la interacción entre el grupo $\mathrm{OH}^{-}$de la HAp y los iones de $\mathrm{Cl}^{-}$de la ClAp (Díaz González, 1974; Han et al., 2021).

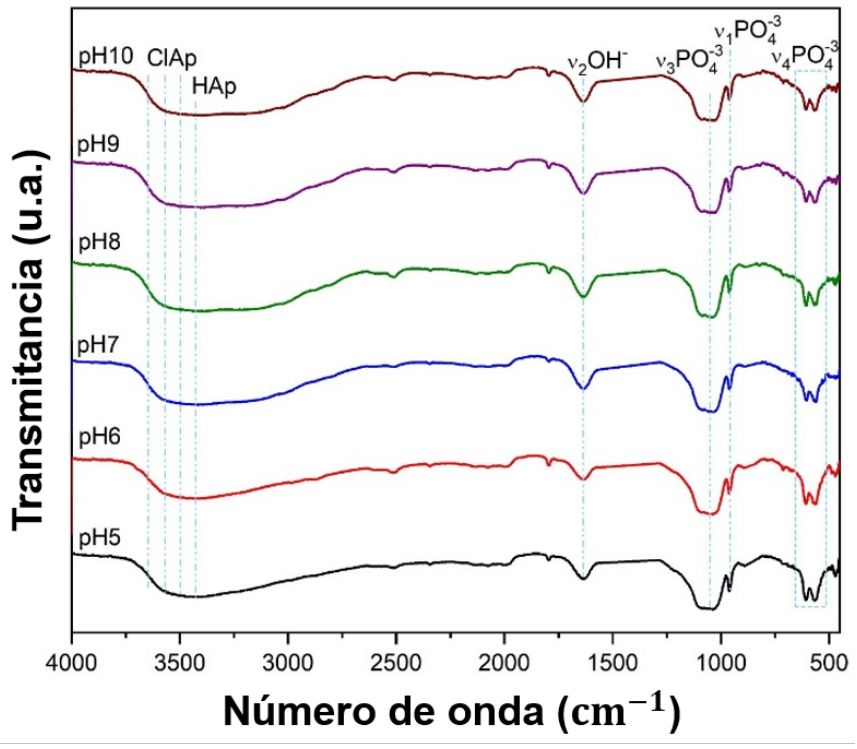

Figura 4. Espectro IR de la HAp y CIAp a pH5, 6, 7, 8, 9 y 10 , respectivamente.

\section{Discusión}

El presente trabajo muestra los resultados obtenidos para la síntesis de hidroxiapatita y cloroapatita sintetizadas por el método hidrotermal asistido por microondas, a partir de cloruro de calcio $\left(\mathrm{CaCl}_{2}\right)$ y fosfato de sodio tribásico dodecahidratado $\left(\mathrm{Na}_{3} \mathrm{PO}_{4} \bullet 12 \mathrm{H}_{2} \mathrm{O}\right)$. Se identificó la variación del tamaño promedio de partícula en función del $\mathrm{pH}$, observando un crecimiento anisotrópico del cristal, formando fibras unidireccionales y algunas estructuras bidimensionales (placas), como se ilustra en la Figura 5. Al tomar como referencia el pH9 (natural), se observa un incremento del diámetro de un $243.54,242.6$ y $250 \%$ para $\mathrm{pH} 8$, 6 y 5 respectivamente, en general representa el incremento de la protonación al disminuir el $\mathrm{pH}$, sin embargo, para el $\mathrm{pH} 7$ se tiene un incremento del $118.2 \%$ y se tiene un aumento del diámetro, menor a los pHs anteriores. Caso similar sucede al incrementar el $\mathrm{pH}$ a 10 , en el cual se aprecia un incremento del $167.2 \%$ con respecto al $\mathrm{pH}$ natural de las muestras. En relación con la longitud de las fibras, se aprecia un comportamiento similar con el diámetro, al tomar como referencia el pH9 (natural), se observa un incremento de $161,199.4$ y $388.8 \%$ en la longitud de las muestras en $\mathrm{pH} 8,6$ y 5 respectivamente. Sin embargo para el pH7 se tiene un incremento del $118.7 \%$, lo que demuestra que incrementa la protonación al disminuir el $\mathrm{pH}$, aunque para el $\mathrm{pH} 7$ se tiene un aumento de la longitud, pero menor a los $\mathrm{pH}$ anteriores, comportamiento que se ve reflejado en el $\mathrm{pH} 10$, ya que presenta un crecimiento de $138.5 \%$ con respecto a las muestras de $\mathrm{pH} 9$ (natural), como consecuencia del uso de hidróxido de amonio al producir una mayor alcalinidad incrementa la formación de $\mathrm{OH}^{-}$ (Ruffini et al., 2019). 


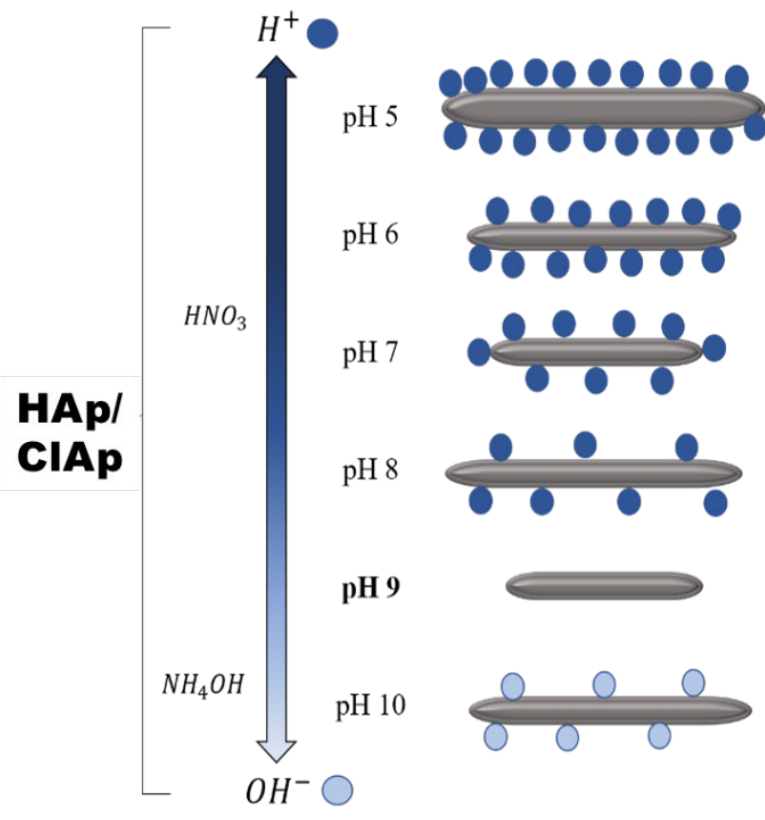

Figura 5. Esquema del efecto del pH en el tamaño promedio de HAp y ClAp a pH 5, 6, 7, 8, 9 y 10 respectivamente.

Por otra parte, el análisis por EDS confirma la presencia de $\mathrm{Ca}, \mathrm{P}$ y $\mathrm{Cl}$. El análisis cuantitativo reveló que la relación de composición de calcio y fósforo es mayor que la relación estequiométrica, de 1.67. Los iones del fosfato fueron substituidos por los iones del carbonato, así la relación de $\mathrm{Ca} / \mathrm{P}$ se incrementa. Esto corrobora investigaciones anteriores, demostrando que la ClAp obtenida es más soluble que la HAp y el ClAp puro, $\mathrm{Ca}_{10}\left(\mathrm{PO}_{4}\right)_{6} \mathrm{Cl}_{2}$, tiene la relación $\mathrm{Ca} / \mathrm{Cl}$ de 5 , lo que indica que a pH5 y pH6, se obtiene la mayor cantidad de cloroapatita (de Almeida Cavalcante et al., 2020; Ito et al., 2017).

El análisis por DRX, muestra la formación de las fases hidroxiapatita y cloroapatita, con una superposición de los picos para ambas fases. Sin embargo, los resultados del análisis del tamaño de cristal arrojan que al aumentar el $\mathrm{pH}$ con hidróxido de amonio de $\mathrm{pH} 9$ a pH 10 el tamaño promedio de cristal disminuye un $4 \%$ de 49 a $47 \mathrm{~nm}$, así mismo, se aprecia una disminución del tamaño promedio de cristal de $10.2,14.2,32.6$ y $36.7 \%$ al emplear ácido nítrico para disminuir el $\mathrm{pH}$ a 8, 7,6 y 5, respectivamente, favoreciendo la formación de fibras largas y tamaños de cristal pequeños.

A partir del análisis por FTIR, se determina la presencia de los grupos funcionales correspondientes a los grupos $\mathrm{OH}^{-}$y $\mathrm{PO}_{4}{ }^{-3}$, atribuidos a HAp y ClAp, así como también, la elongación situada en la región de las vibraciones del grupo $\mathrm{OH}^{-}, 3570$ y $3498 \mathrm{~cm}^{-1}$. El primer modo vibracional ocupa la posición análoga a la de la banda $\mathrm{OH}^{-}$de la HAp, esta es originada por los grupos $\mathrm{OH}^{-}$que no interaccionan con el cloro; el segundo modo vibracional se debe a los $\mathrm{OH}^{-}$de la HAp unidos por puente de hidrógeno con dichos iones $\mathrm{Cl}^{-}$de la $\mathrm{ClAp}$, en $3640 \mathrm{~cm}^{-1}$ se atribuye al modo vibracional de los grupos $\mathrm{OH}^{-}$situados en el exterior de la estructura, debido al desplazamiento por la introducción de los iones de $\mathrm{Cl}^{-}$a la estructura, pareciera que no existen iones $\mathrm{Cl}^{-}$en estas posiciones; sin embargo, para el caso de la banda situada en $3640 \mathrm{~cm}^{-1}$, se debe tener en cuenta que la introducción de los iones $\mathrm{Cl}^{-}$en el interior de la estructura provoca la salida de los grupos $\mathrm{OH}^{-}$al exterior, explica el porqué de la presencia de esta banda en la región $\mathrm{OH}^{-}$. Para el segundo caso se debe a la unión por puentes de hidrógeno entre los $\mathrm{OH}^{-}$ de la HAp y los iones $\mathrm{Cl}^{-}$de la $\mathrm{ClAp}$, lo que confirma la formación de ambas fases.

Los resultados obtenidos permiten determinar el efecto del $\mathrm{pH}$; al disminuir de $\mathrm{pH} 9$ (natural) a 8, 7,6 y 5, implica la generación de una protonación, es decir, de un incremento de $\mathrm{H}^{+}$, y para incrementa de pH9 a 10, se genera la formación de $\mathrm{OH}^{-}$, identificando que para ambos casos se incrementa el tamaño del diámetro y longitud de la estructura, favoreciendo el crecimiento de partículas en forma de fibras con tamaño de cristales pequeños. A continuación, se muestra una tabla comparativa de los trabajos reportados en la literatura similares a esta investigación (Tabla 3):

Tabla 3. Tabla comparativa de trabajos similares reportados en la literatura.

\begin{tabular}{|c|c|c|c|}
\hline $\mathrm{pH}$ & Fases obtenidas & Morfología & Dimensiones \\
\hline $\begin{array}{c}7,8,9 \text { y } 10 \\
\text { (Sánchez-campos } \\
\text { et al., 2021) }\end{array}$ & $\begin{array}{c}\text { HAp } \\
\text { (hexagonal y } \\
\text { monoclínica) }\end{array}$ & Nanobarras & $\begin{array}{c}29-60 \mathrm{~nm} \text { de } \\
\text { diámetro y } \\
81-106 \mathrm{~nm} \text { de } \\
\text { longitud. }\end{array}$ \\
\hline $\begin{array}{c}8 \text { a } 10 \\
\text { (Goh et al., 2021) }\end{array}$ & HAp & Aguja & $\begin{array}{c}10-15 \mathrm{~nm} \text { de } \\
\text { diámetro y } \\
60-80 \mathrm{~nm} \text { de } \\
\text { longitud. }\end{array}$ \\
\hline $\begin{array}{c}8,10 \text { y } 13 \\
\text { (Yudin et al., } \\
\text { 2019) }\end{array}$ & HAp & Irregular & $\begin{array}{c}\text { Aglomerados } \\
(0.5-25 \mu \mathrm{m}), \\
\text { formado por } \\
\text { partículas de } \\
17-46 \mathrm{~nm} \text {. }\end{array}$ \\
\hline $\begin{array}{c}7,9 \text { y } 11 \\
\text { (Amalia et al., } \\
2020)\end{array}$ & HAp y ClAp & Redondeadas & $60-180 \mathrm{~nm}$ \\
\hline
\end{tabular}

Por lo tanto, los resultados obtenidos en este trabajo demuestran que al variar el $\mathrm{pH}$ de la solución, se pueden obtener morfologías regulares en forma de barras dentro de una escalas micrométrica y nanométrica mediante el método hidrotermal asistido por microondas, que permite la obtención de estos productos en un menor tiempo de síntesis (40 min), logrando un control en el tamaño y forma, sin necesidad de emplear agentes de superficie activa, como lo reportan en otros trabajos, debido a que el $\mathrm{pH}$ es un parámetro de reacción el cual se encuentra directamente relacionado con la fase cristalina, morfología y tamaños obtenidos.

\section{Conclusiones}

A partir de los resultados obtenidos se demuestra que es posible obtener de manera simultánea dos apatitas con aplicaciones biomédicas: hidroxiapatita y cloroapatita. El primero ampliamente utilizado como relleno en huesos y dientes, mientras que el segundo tiene la capacidad para desarrollar un ambiente ácido en la superficie del hueso que activa los osteoclastos en el proceso de resorción ósea. También se encontró que las características morfológicas y estructurales de ambas apatitas dependen del $\mathrm{pH}$ con el que se inicia la reacción, lo que permite establecer el control de tamaño y forma del material, el cual puede ser empleado con fines para el desarrollo de biomateriales. Sin embargo, el trabajo de investigación se debe de profundizar con el propósito de lograr una relación $\mathrm{Ca} / \mathrm{P}$ de 1.67 , que es la estequiométrica, como consecuencia de que en el rango de 1.5 a 1.7 se favorece la formación de hueso y a medida que se disminuye la relación $\mathrm{Ca} / \mathrm{P}$ se incrementa la solubilidad y la bioabsorción. Sin embargo, en los materiales que poseen una relación $\mathrm{Ca} / \mathrm{P}$ menores a 1 la bioadsorción se lleva aún más rápido para implantación de tejido, asimismo una relación $\mathrm{Ca} / \mathrm{P}$ mayor a 
2, presenta basicidad y aumenta la insolubilidad, por lo que no son adecuados para implantación en el cuerpo.

\section{Agradecimientos}

Agradecimiento del autor al CONACYT por el apoyo a Lesly Sabina Villaseñor Cerón y Susana López Ortiz por la beca otorgada en el doctorado (0432) perteneciente al Área Académica de Ciencias de la Tierra y Materiales e Instituto de Ciencias Básicas e Ingeniería de la Universidad Autónoma del Estado de Hidalgo.

\section{Referencias}

Amalia, T. R., Nurlely, \& Sari, Y. W. (2020). Synthesis of Hidroxyapatite Using Microwave Irradiation and Sintering with Variation $\mathrm{pH}$ and Time. Journal of Physics: Conference Series, 1505(1). https://doi.org/10.1088/1742$6596 / 1505 / 1 / 012063$

Chetty, A. S., Wepener, I., Marei, M. K., El, Y., \& M., R. (2012). Synthesis, Properties, and Applications of Hydroxyapatite. In V. S. Gshalaev and A. C. Demirchan (Ed.), Hydroxyapatite: Synthesis, Properties and Applications (pp. 91-134). Nova Science Publishers, Inc.

Ciobanu, C. S., Iconaru, S. L., Pasuk, I., Vasile, B. S., Lupu, A. R., Hermenean, A., Dinischiotu, A., \& Predoi, D. (2013). Structural properties of silver doped hydroxyapatite and their biocompatibility. Materials Science and Engineering: C, 33(3), 1395-1402. https://doi.org/10.1016/J.MSEC.2012.12.042

de Almeida Cavalcante, L., Ribeiro, L. S., Takeno, M. L., Pandava Aum, P. T., Aum, Y. K. P. G., \& Silva Andrade, J. C. (2020). Chlorapatite derived from fish scales. Materials, 13(5), 1-11. https://doi.org/10.3390/ma13051129

Deng, R., Huang, D., Xue, W., Lei, L., Chen, S., Zhou, C., Liu, X., Wen, X., \& Li, B. (2020). Eco-friendly remediation for lead-contaminated riverine sediment by sodium lignin sulfonate stabilized nano-chlorapatite. Chemical Engineering Journal, 397(April). https://doi.org/10.1016/j.cej.2020.125396

Díaz González, P. F. (1974). Cloroapatita. In Estudio por espectroscopía infrarroja de hidroxilapatitas y derivados.

Dorozhkin, S. V. (2010). Nanosized and nanocrystalline calcium orthophosphates. In Acta Biomaterialia (Vol. 6, Issue 3, pp. 715-734). https://doi.org/10.1016/j.actbio.2009.10.031

Ferraz, M. P., Monteiro, F. J., \& Manuel, C. M. (2018). Hydroxyapatite nanoparticles: A review of preparation methodologies. Journal of Applied Biomaterials \& Biomechanics: $\quad J A B B, \quad 2(2), \quad 74-80$. https://doi.org/10.1177/228080000400200202

Goh, K. W., Wong, Y. H., Ramesh, S., Chandran, H., Krishnasamy, S., Sidhu, A., \& Teng, W. D. (2021). Effect of $\mathrm{pH}$ on the properties of eggshell-derived hydroxyapatite bioceramic synthesized by wet chemical method assisted by microwave irradiation. Ceramics International, 47(7), 8879-8887.

https://doi.org/10.1016/j.ceramint.2020.12.009

Habibovic, P., Kruyt, M. C., Juhl, M. V., Clyens, S., Martinetti, R., Dolcini, L., Theilgaard, N., \& Van Blitterswijk, C. A. (2008). Comparative in vivo study of six hydroxyapatitebased bone graft substitutes. Journal of Orthopaedic
Research,

$26(10)$

https://doi.org/10.1002/jor.20648

$1363-1370$

Han, X., Zhang, Y., Zheng, C., Yu, X., Li, S., \& Wei, W. (2021). Enhanced $\mathrm{Cr}(\mathrm{VI})$ removal from water using a green synthesized nanocrystalline chlorapatite: Physicochemical interpretations and fixed-bed column mathematical model study. Chemosphere, 264, 128421. https://doi.org/10.1016/j.chemosphere.2020.128421

Harlov, D. E., Förster, H. J., \& Nijland, T. G. (2002). Fluidinduced nucleation of (Y + REE)-phosphate minerals within apatite: Nature and experiment. Part I. Chlorapatite. American Mineralogist, 87(2-3), 245-261. https://doi.org/10.2138/am-2002-2-306

Huang, D., Deng, R., Wan, J., Zeng, G., Xue, W., Wen, X., Zhou, C., Hu, L., Liu, X., Xu, P., Guo, X., \& Ren, X. (2018). Remediation of lead-contaminated sediment by biocharsupported nano-chlorapatite: Accompanied with the change of available phosphorus and organic matters. Journal of Hazardous Materials, 348, 109-116. https://doi.org/10.1016/j.jhazmat.2018.01.024

Ito, A., Otsuka, Y., Takeuchi, M., \& Tanaka, H. (2017). Mechanochemical synthesis of chloroapatite and its characterization by powder X-ray diffractometory and attenuated total reflection-infrared spectroscopy. Colloid and Polymer Science, 295(10), 2011-2018. https://doi.org/10.1007/s00396-017-4177-7

Kannan, S., Rebelo, A., Lemos, A. F., Barba, A., \& Ferreira, J. M. F. (2007). Synthesis and mechanical behaviour of chlorapatite and chlorapatite/ $\beta$-TCP composites. Journal of the European Ceramic Society, 27(5), 2287-2294. https://doi.org/10.1016/j.jeurceramsoc.2006.07.004

Kokubo, T., \& Takadama, H. (2006). How useful is SBF in predicting in vivo bone bioactivity? Biomaterials, 27(15), 2907-2915.

https://doi.org/10.1016/j.biomaterials.2006.01.017

Koutsopoulos, S. (2002). Synthesis and characterization of hydroxyapatite crystals: A review study on the analytical methods. Journal of Biomedical Materials Research, 62(4), 600-612. https://doi.org/10.1002/jbm.10280

Kusakabe, H., Sakamaki, T., Nihei, K., Oyama, Y., Yanagimoto, S., Ichimiya, M., Kimura, J., \& Toyama, Y. (2004). Osseointegration of a hydroxyapatite-coated multilayered mesh stem. Biomaterials, 25(15), 2957-2969. https://doi.org/10.1016/J.BIOMATERIALS.2003.09.090

López-Ortiz, S., Mendoza-Anaya, D., Sánchez-Campos, D., Fernández-García, M. E., Salinas-Rodríguez, E., ReyesValderrama, M. I., \& Rodríguez-Lugo, V. (2020). The pH Effect on the Growth of Hexagonal and Monoclinic Hydroxyapatite Synthesized by the Hydrothermal Method. Journal of Nanomaterials, 2020. https://doi.org/10.1155/2020/5912592

Mehdi Sadat-Shojai, Mohammad-Taghi Khorasani, Ehsan Dinpanah-, Khoshdargi, \& Ahmad Jamshidi. (2013). Synthesis methods for nanosized hydroxyapatite with diverse structures. Acta Biomaterialia, 9(8), 7591-7621. https://doi.org/10.1016/J.ACTBIO.2013.04.012

O'Hare, P., Meenan, B. J., Burke, G. A., Byrne, G., Dowling, D., \& Hunt, J. A. (2010). Biological responses to hydroxyapatite surfaces deposited via a co-incident microblasting technique. Biomaterials, 31(3), 515-522. https://doi.org/10.1016/j.biomaterials.2009.09.067

Olszta, M. J., Cheng, X., Jee, S. S., Kumar, R., Kim, Y.-Y., Kaufman, M. J., Douglas, E. P., Gower, L. B., \& Gower, L. B. (2007). Bone structure and formation: A new perspective. Materials Science and Engineering $R, 58,77-$ 
116. https://doi.org/10.1016/j.mser.2007.05.001

Panda, R. N., Hsieh, M. F., Chung, R. J., \& Chin, T. S. (2003). FTIR, XRD, SEM and solid state NMR investigations of carbonate-containing hydroxyapatite nano-particles synthesized by hydroxide-gel technique. Journal of Physics and Chemistry of Solids, 64(2), 193-199. https://doi.org/10.1016/S0022-3697(02)00257-3

Rabiei, A., Blalock, T., Thomas, B., Cuomo, J., Yang, Y., \& Ong, J. (2007). Microstructure, mechanical properties, and biological response to functionally graded HA coatings. Materials Science and Engineering C, 27(3), 529-533. https://doi.org/10.1016/j.msec.2006.05.036

Recillas, S., Mondragón-Galicia, G., Rodríguez-Lugo, V., \& Castaño, V. M. (2003). Growth of calcium phosphate onto chemically-functionalized cottons. Designed Monomers and Polymers, 6(4), 383-398. https://doi.org/10.1163/156855503771816840

Rodríguez-Lugo, V., Angeles-Chavez, C., \& Hernandez, M. (2003). Synthesis of hydroxylapatite from sand dollar and $\beta$-tricalcium phosphate by solid-state method. Materials and Manufacturing Processes, 18(6), 903-913. https://doi.org/10.1081/AMP-120025078

Rodríguez-Lugo, V., Angeles, C., De la Isla, A., \& Castano, V. M. (2015). Effect of bio-calcium oxide on the morphology of hydroxyapatite. International Journal of Basic and Applied Sciences, 4(4), 395. https://doi.org/10.14419/ijbas.v4i4.5240

Rodríguez-Lugo, V., Ascencio, J. A., Angeles-Chavez, C., Camacho-Bragado, A., \& Castaño, V. M. (2001). Controlled Hydrothermal Production Of Hydroxylapatite From Marine Skeletons. Materials and Technology, 16(2), 97-103. https://doi.org/10.1080/10667857.2001.11752918

Rodríguez-Lugo, V., Karthik, T. V. K., Mendoza-Anaya, D., Rubio-Rosas, E., Villaseñor Cerón, L. S., ReyesValderrama, M. I., \& Salinas-Rodríguez, E. (2018). Wet chemical synthesis of nanocrystalline hydroxyapatite flakes: effect of $\mathrm{pH}$ and sintering temperature on structural and morphological properties. Royal Society Open Science, 5(8), 180962. https://doi.org/10.1098/rsos.180962

Rodríguez-Lugo, V., Mendoza-Anaya, D., \& Estrada, R. F. (2012). Biomimetic Growth of Hydroxyapatite on Collagen. Revista Electrónica Ideas CONCYTEG, 7(87), $1127-1136$

Rodríguez-Lugo, V., Salado-Leza, D. E., López Ortiz, S., Mendoza-Anaya, D., Villaseñor-Cerón, L. S., \& ReyesValderrama, M. I. (2020). Revisión de la Hidroxiapatita Nanoestructurada como Alternativa para Tratamiento de Cáncer. Pädi Boletín Científico de Ciencias Básicas e Ingenierías Del ICBI, 8(Especial), 115-127. https://doi.org/10.29057/icbi.v8iespecial.6466

Ruffini, A., Sprio, S., \& Preti, L. (2019). Synthesis of Nanostructured Hydroxyapatite via Controlled
Hydrothermal Route. In Biomaterial-supported Tissue Reconstruction or Regeneration. https://doi.org/10.5772/intechopen.77756

Rulis, P., Ouyang, L., \& Ching, W. Y. (2004). Electronic structure and bonding in calcium apatite crystals: Hydroxyapatite, fluorapatite, chlorapatite, and bromapatite. Physical Review $B$ - Condensed Matter and Materials Physics, 70(15), 1-8. https://doi.org/10.1103/PhysRevB.70.155104

Sáenz, A., Montero, M. L., Mondragón, G., Rodríguez-Lugo, V., \& Castaño, V. M. (2003). Effect of $\mathrm{pH}$ on the precipitation of hydroxyapatite on silica gels. Materials Research Innovations, 7(2), 68-73. https://doi.org/10.1007/s10019003-0230-X

Sánchez-Campos, D., Reyes-Valderrama, M. I.., López-Ortíz, S., Salado-leza, D., Fernández-García, M. E., Mendoza-Anaya, D., Salinas-Rodríguez, E., \& Rodríguez-Lugo, V. (2021). Modulated monoclinic hydroxyapatite: The effect of ph in the microwave assisted method. Minerals, 11(3), 1-13. https://doi.org/10.3390/min 11030314

Szcześ, A., Hołysz, L., \& Chibowski, E. (2017). Synthesis of hydroxyapatite for biomedical applications. https://doi.org/10.1016/j.cis.2017.04.007

Wan, J., Zeng, G., Huang, D., Hu, L., Xu, P., Huang, C., Deng, R., Xue, W., Lai, C., Zhou, C., Zheng, K., Ren, X., \& Gong, $X$. (2018). Rhamnolipid stabilized nano-chlorapatite: Synthesis and enhancement effect on $\mathrm{Pb}$-and $\mathrm{Cd}$ immobilization in polluted sediment. Journal of Hazardous Materials, 343, 332-339. https://doi.org/10.1016/j.jhazmat.2017.09.053

Yanagisawa, K., Rendon-Angeles, J. C., Ishizawa, N., \& Oishi, S. (1999). Topotaxial replacement of chlorapatite by hydroxyapatite during hydrothermal ion exchange. American Mineralogist, 84(11-12), 1861-1869. https://doi.org/10.2138/am-1999-11-1213

Yudin, A., Ilinykh, I., Chuprunov, K., Kolesnikov, E., Kuznetsov, D., Leybo, D., \& Godymchuk, A. (2019). Microwave treatment and $\mathrm{pH}$ influence on hydroxyapatite morphology and structure. Journal of Physics: Conference Series, $1145(1)$. $6596 / 1145 / 1 / 012003$

Zheo, J., Dong, X., Bian, M., Zhao, J., Zhang, Y., Sun, Y., Chen, J., \& Wang, X. (2014). Solution combustion method for synthesis of nanostructured hydroxyapatite, fluorapatite and chlorapatite. Applied Surface Science, 314, 1026-1033. https://doi.org/10.1016/j.apsusc.2014.06.075

Zuo, G., Wei, X., Sun, H., Liu, S., Zong, P., Zeng, X., \& Shen, Y. (2017). Morphology controlled synthesis of nanohydroxyapatite using polyethylene glycol as a template. Journal of Alloys and Compounds, 692, 693-697. https://doi.org/10.1016/j.jallcom.2016.09.117 